\title{
THROUGHPUT IMPROVEMENT UTILIZING NOVEL PROPOSED MODEL COMBINING BCH (N, K) CODE WITH STBC CODE OVER MIMO SYSTEM WITH RAYLEIGH FADING CHANNEL
}

\section{${ }^{*}$ Muntadher Suhail Abed ${ }^{1}$}

Dr. Raad H.Thaher ${ }^{2}$

1) M.Sc. Student, Electrical Engineering Dep., Al-Mustansiriyah University College of Engineering, Baghdad, Iraq.

2) Prof. Dr., Electrical Engineering Dep., Al-Mustansiriyah University College of Engineering، Baghdad, Iraq.

\begin{abstract}
Several coding schemes have been implemented to improve capacity (throughput), minimize bit error rate, and the effect of fading. MIMO technique is stilling a powerful technique besides channel coding. The structure of the proposed system model relies on channel coding represented by $\mathrm{BCH}(n, k)$ code combined with MIMO-STBC code. In this research paper, focus on utilizing accessible wireless communication resources to improve the efficiency of the MIMO system in terms of capacity (throughput) and coding gain utilizing the proposed model combining $\mathrm{BCH}(\mathrm{n}, \mathrm{k})$ code with STBC code over MIMO system with Rayleigh fading channel. Therefore, the outcomes achieved by the proposed model gave a better improvement in coding gain and capacity (throughput). Relative to $\mathrm{BCH}(31,26)$, the improvement in coding gain about $6.94 \mathrm{~dB}$ and achieved even better improvement if increase length of $\mathrm{BCH}$ code. Thus, for $\mathrm{BCH}(31,11)$, the improvement in coding gain about $16.9 \mathrm{~dB}$ at BER $\left(10^{-3}\right)$. Additionally, the improvement in system performance reaches (65\%$100 \%$ ). This work is executed utilizing MATLAB simulation. The findings are analyzed and compared.
\end{abstract}

Keywords: $M I M O, B C H$ code, coding gain, throughput, SNR, and Rayleigh fading channel.

\section{Introduction}

Owing to an ever-growing need for efficient and reliable digital transmission and growing demands for requirements such as BER, service efficiency, and increasing the number of communication service users [1].Modern technologies have demonstrated tremendous communication demands with insufficient transmission power and bandwidth constraints, which, in effect, will not allow us to develop all services[2]. So, finding a suitable strategy for these determinants is a significant challenge.

The efficiency of all wireless communications systems relies on the channel environment. The wireless channel is, therefore, rather complex and unpredictable in design. Furthermore, the fading and shadowing of multipath are primarily limited to decreasing system quality. Therefore, to obtain high spectral performance, several antennas must be used at transmitting and receiving ends. In this case, the multiple-input multiple-output system (MIMO) is a possible nominee for the $5 \mathrm{G}$ communication system[3].MIMO systems have become very common in wireless communication, especially LTE and Wi-Fi [4-5]. MIMO technology uses many antennas at the transmit and receive to provide several benefits preferable over single input - single output (SISO) system[3]. Coding

*Corresponding Author: muntadhersuhail@gmail.com 
gain calculates the difference in SNR level among the coded and un-coded systems at a specified error rate [6].

Exploiting the MIMO strategy in communication regimes has two primary advantages: diversity and multiplexing. Through multiplexing, the transmitting rate has been increased, and the communication reliability of the system is enhanced via exploit space and time diversity[7]. Space-time block code (STBC) is a sufficient transmit diversity employed to contend drawback effects of fading in wireless channels due to its easy decoding and realizing full diversity and highest throughput[8].

Rayleigh fading channel (NLOS) is primarily produced by multipath reception when there is a large number of paths via the transmission channel that consists of the received signal at the recipient side. It is an appropriate model for the troposphere and ionosphere signals propagation. The impulse response of such a channel model will have a Gaussian distribution if a central limit theorem is adopted. When the process has a zero mean, the channel response envelope at any time will have a Rayleigh distribution PDF, and the phase will be distributed uniformly over the period $(0,2 \pi)[9$ 10].

Binary phase-shift keying modulation has been two-state phase shift keying in which the phase of the carrier is set to 0 or $\pi$ depending on the modulating signal values. The modulation signal was a binary series and is multiplied via a sinusoidal carrier, which produces the modulated BPSK signal. Hence, When the symbol "1" was sent, the modulated signal is almost the same as the carrier with the $0^{\circ}$ initial phase. The modulated signal gets a $180^{\circ}$ initial phase carrier burst while "0" is sent[11].
In this respect, different research has been done to improve the performance of the MIMO system utilizing coding strategies attempt to get a perfect combination given enhancement in channel capacity with low complexity and maximum coding gain.

In 2012, Abdul Kareem Abdurrahman [12] introduced a study involved modern methodology for achieving the best system performance utilizing the MIMO system and network coding with various types of channels. The outcomes demonstrated that combining MIMO technique and network coding lead to enhancement BER and throughput of the system.

In 2013 S. Zhao [13], presented a study involved concatenation scheme of polar codes and STBC codes over MIMO system, this concatenation is called ( Polar-STBC) scheme, the outcomes showed this concatenation give significant enhancement in BER performance of MIMO system as when compared with the system of STBC only and achieved coding gain about $5 \mathrm{~dB}$ at BER $\left(10^{-3}\right)$ and when incrementing the length of Polar codes, the BER performance of (polar -STBC) scheme is as well improved too.

In 2015, Elise Ghayoula [14], introduced a study involving the proposed concatenation of low-density parity-check(LDPC code) with STBC code over the MIMO system. The outcomes showed these concatenation improvements the performance of the MIMO system and achieved coding gain about $9 \mathrm{~dB}$ at BER $\left(2 * 10^{-4}\right)$ as when compares with STBC only.

In this research paper, demonstrating throughput improvement utilizing the proposed model combining BCH (n, k) code with STBC code over the MIMO system and compares with un-coded state over Rayleigh fading channel 
and exploited Binary phase shift keying as a modulation scheme.

The residue of this paper is organized as follows. In section II, $\mathrm{BCH}$ codes. In section. III, the proposed model. In section IV, Simulation results were described. And eventually, in section $\mathrm{V}$, conclusions and future works.

\section{BCH Codes}

$\mathrm{BCH}$ codes are a new subclass of cyclic errorcorrecting code, discovered by (Bose- RayChaudhuri-Hocquenghem) [15-16]. An essential aspect of this code, it is simple to encode and easy to decode the signal. Comparable to modernist coding techniques such as LDPCs and turbo codes, the $\mathrm{BCH}$ decoder is lower complexity and reliable bug control over symbol error numbers [17]. The properties of this code illustrate in Table 1.

Table 1. Properties of BCH code[15]

\begin{tabular}{cc}
\hline Type & Linear block code \\
Alphabet Size & 2 \\
Parameter & $(\mathrm{n}, \mathrm{k}, \mathrm{d})_{2}$ \\
Number of corrected error $(\mathrm{t})$ & $\mathrm{t}$ where $\mathrm{t}<2^{\mathrm{m}}-1$ \\
Block length $(\mathrm{n})$ & $2^{\mathrm{m}}-1$ \\
Massage length $(\mathrm{k})$ & $\mathrm{k}$ \\
Code rate $(\mathrm{R})$ & $\mathrm{k} / \mathrm{n}$ \\
Distance $(\mathrm{d})$ & $\mathrm{d}$
\end{tabular}

Code-word being generated by dividing the $\mathrm{m}(\mathrm{x})$ polynomial expressing the data (binary data) by $\mathrm{g}(\mathrm{x})$ polynomial generator and take the remainder that is described as parity check bits $\mathrm{r}(\mathrm{x})$. Code specifications are determined by selecting a polynomial generator $\mathrm{g}(\mathrm{X})[18]$. The generator polynomial $\mathrm{g}(\mathrm{x})$ could be calculated employing the following equation:
$\mathrm{g}(\mathrm{X})=\operatorname{LCM}\left[\Phi_{1}(\mathrm{X}), \Phi_{3}(\mathrm{X}), \ldots, \Phi_{2 \mathrm{t}-1}(\mathrm{X})\right]$

Where LCM refers to the lowest common multiple. The minimal polynomial ( $\alpha \mathrm{i}$ ) represent by $\Phi \mathrm{i}(\mathrm{X})$, and can be calculated by the following formula [19]:

$\Phi \mathrm{i}(\mathrm{X})=\Pi\left(\mathrm{X}+\alpha^{\mathrm{j}}\right)$

Due to $g(X)$ is calculated from the production of $\mathrm{t}$ minimal polynomial for degrees about $\mathrm{m}$. The maximum degree of $\mathrm{g}(\mathrm{x})$ is $(\mathrm{m} * \mathrm{t})$. its implies the parity check digit (n-k) is almost equal to $\left(m^{*} t\right)$.

The encoding process of $\mathrm{BCH}$ code taking the massage block of length $(\mathrm{k})$ and convert it to the code word with length (n). The following steps can explain the encoding procedure:

- Multiplying the message $\mathrm{m}(\mathrm{x})$ by $\left(X^{n-k}\right)$, this multiplication is equal to a shift of the message bits though $(\mathrm{n}-\mathrm{k})$ to the right.

- Calculate remaining $\mathrm{r}(\mathrm{x})$ from division the $\left(X^{n-k} m(x)\right)$ by the $g(X)$ polynomial generator. The remainder is the parity check digits.

- In order to obtain code-word polynomial $\mathrm{C}(\mathrm{x})$, adding the calculated residual $\mathrm{r}(\mathrm{x})$ to $\left(\left(X^{n-k} m(x)\right)\right.$.

This method as well guarantees that the code word in methodical form, which means that the data bits described by the polynomial $\mathrm{m}(\mathrm{x})$ can be conveniently interpreted the code-word polynomial $\mathrm{c}(\mathrm{x})$ by a look at the rightmost $\mathrm{k}$ coefficient.

Now, employing three steps to achieve the decoding process of $\mathrm{BCH}$ code described as follow:

- Syndrome $S=\left(S_{1}, S_{2}, S_{3}, \ldots \ldots S_{2 t}\right)$ was determined via code-word received $\mathrm{r}(\mathrm{x})$. 
- Error location in the polynomial $\sigma(\mathrm{x})$ is calculated employing syndrome-derived equations $S_{2 t}$.

- Polynomial error location was employed for defining and correcting erroneous bits.

For $(\mathrm{t})$ error correction code, the syndrome $(\mathrm{S})$ composed of $2 t$ is called $S 1, S_{2}, S_{3} \ldots S_{2 t}$. The components of the syndrome can be determined by employing the following equations:

$$
S_{i}=r\left(\alpha^{i}\right)
$$

In order to acquire the code-word polynomial $\mathrm{e}(\mathrm{x})$, addition the polynomial error pattern $\mathrm{e}(\mathrm{x})$ with sent polynomial code-word $\mathrm{c}(\mathrm{x})$, and can be expressed as:

$r(x)=c(x)+e(x)$

Because each polynomial code-word should have the roots $\alpha$. The components of syndrome rely solely on the polynomial error pattern $\mathrm{e}(\mathrm{x})$. Consequently, we could be express as:

$\mathrm{S}_{\mathrm{i}}=\mathrm{e}\left(\alpha^{\mathrm{i}}\right)$

Assume that $\mathrm{e}(\mathrm{x})$ error patterns have $\mathrm{v}$ errors at the location $\mathrm{X}^{\mathrm{j} 1}, \mathrm{X}^{\mathrm{j} 2}, \mathrm{X}^{\mathrm{j} 3} \ldots \ldots . \mathrm{X}^{\mathrm{jv}}$. Can be express as:

$e(X)=X^{j 1}+X^{j 2}+X^{j 3}+\ldots \ldots \ldots+X^{j v}$

From equation (5) and (6), acquired the following equations:

$S_{1}=\alpha^{j 1}+\alpha^{j 2}+\alpha^{j 3}+\ldots \ldots+\alpha^{j v}$

$S_{2}=\left(\alpha^{j 1}\right)^{2}+\left(\alpha^{j 2}\right)^{2}+\ldots .+\left(\alpha^{j v}\right)^{2}$

$S_{3}=\left(\alpha^{j 1}\right)^{3}+\left(\alpha^{j 2}\right)^{3}+\ldots+\left(\alpha^{j v}\right)^{3}$

$S_{2 t}=\left(\alpha^{j 1}\right)^{2 t}+\left(\alpha^{j 2}\right)^{2 t}+\cdots+\left(\alpha^{j v}\right)^{2 t}$

Where $0 \leq j_{1}<j_{2}<j_{3} \ldots .<j_{v}<n$, and $\alpha^{j 1}, \alpha^{j 2}, \alpha^{j 3} \ldots \ldots, \alpha^{j v}$ are not defined. Thus, any approach to solving such equations is a $\mathrm{BCH}$ code decoding algorithm [19].

\section{The Proposed Model}

In this section, demonstrates the proposed model in Fig.1.

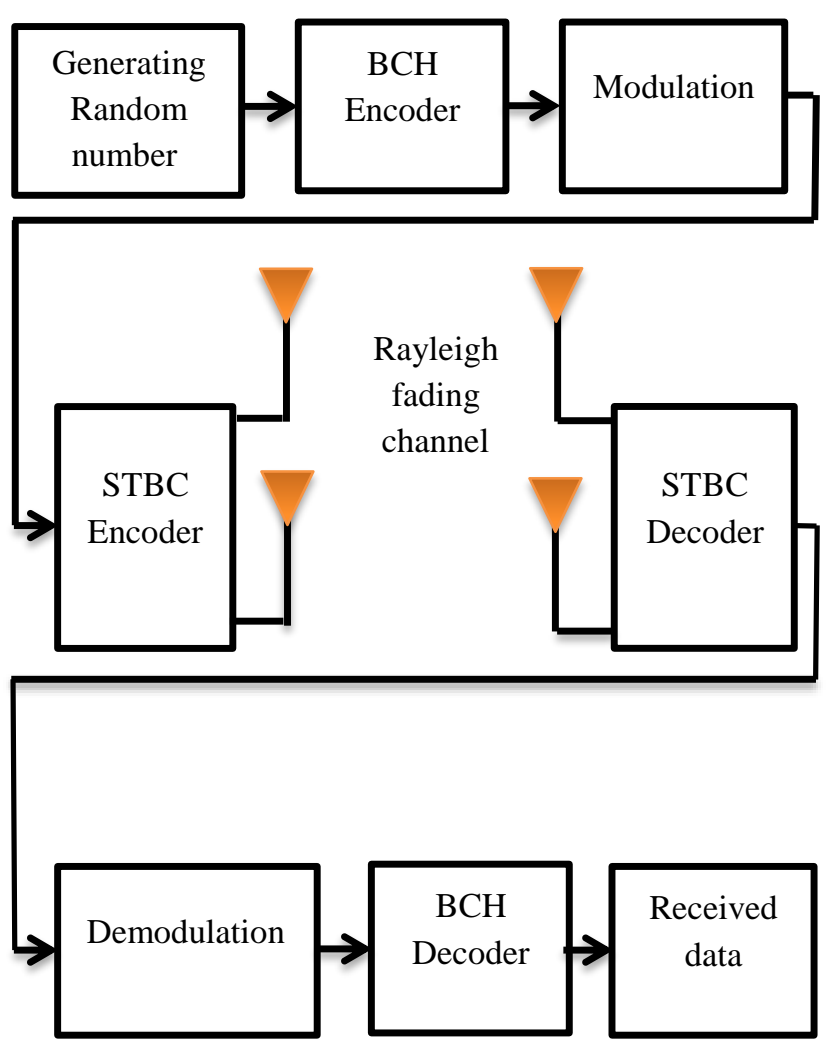

Figure 1.The proposed model

The data (binary data) encoded through the $\mathrm{BCH}$ encoder block, and the code-words $\mathrm{n}$ bit is modulation in the modulated block (Binary phase-shift keying is employed for modulation) to the sent symbol that will be $(\mathrm{S})$ to STBC encoder later. In the resultant STBC code matrix, the data is sent via the antenna of transmitting. In the receiving part, the data from the obtained multiple antennas is estimated by channel estimation and merge by combined of a signal. The maximum likelihood decoding algorithm is used to illustrate the STBC merger signal. Eventually, decoded the data by the $\mathrm{BCH}$ decoder.

In the proposed model, the STBC code offers spatial diversity in the form of MIMO systems to ensure safe wireless communication at the high 
data rate. STBC must be employed to assure orthogonality at the receiver, the received signal is shown:

$$
\begin{gathered}
{\left[\begin{array}{ll}
y_{11} & y_{12} \\
y_{21} & y_{22}
\end{array}\right]=\left[\begin{array}{ll}
h_{11} & h_{12} \\
h_{21} & h_{22}
\end{array}\right]\left[\begin{array}{cc}
s_{1} & -s_{2}^{*} \\
s_{2} & s_{1}^{*}
\end{array}\right]+} \\
{\left[\begin{array}{ll}
N_{11} & N_{12} \\
N_{21} & N_{22}
\end{array}\right]}
\end{gathered}
$$

Where

$\mathrm{s}_{1}, \mathrm{~s}_{2}$ : are the data transmitted.

$\mathrm{y}_{11}, \mathrm{y}_{12}$ : are data received for the 1st-time slot.

$\mathrm{y}_{21}, \mathrm{y}_{22}$ : are data received for the 2nd-time slot.

(*): refers to conjugate.

$\mathrm{h}_{11}, \mathrm{~h}_{12}, \mathrm{~h}_{21}, \mathrm{~h}_{22}$ : Are Rayleigh channel.

$\mathrm{N}_{11}, \mathrm{~N}_{12}, \mathrm{~N}_{21}, \mathrm{~N}_{22}$ : are additive white Gaussian noise (AWGN).

$$
\begin{aligned}
& y_{11}=h_{11} s_{1}+h_{12} s_{2}+N_{11} \\
& y_{12}=-h_{11} s_{2}^{*}+h_{12} s_{1}^{*}+N_{12} \\
& y_{21}=h_{21} s_{1}+h_{22} s_{2}+N_{21} \\
& y_{22}=-h_{21} s_{2}^{*}+h_{22} s_{1}^{*}+N_{22}
\end{aligned}
$$

Assume:

$$
k=\left|h_{11}\right|^{2}+\left|h_{12}\right|^{2}+\left|h_{21}\right|^{2}+\left|h_{22}\right|^{2}
$$

After the STBC decoder, therefore, the data transmitted at the receiver with channel effect and noise.

$$
\begin{gathered}
\left.\tilde{s}_{1}=\frac{1}{K} \begin{array}{lll}
h_{11}^{*} & y_{11}+h_{12} y_{12}+h_{12}^{*} y_{21}+ \\
h_{22} & y_{22}
\end{array}\right] \\
\tilde{s}_{2}=\frac{1}{K}\left[\begin{array}{lll}
h_{12}^{*} & y_{11}-h_{12} y_{12}+h_{22}^{*} y_{21}+ \\
h_{22} & y_{22}
\end{array}\right]
\end{gathered}
$$

\begin{tabular}{|c|c|c|c|c|}
\hline \multirow[t]{2}{*}{ No } & \multirow[t]{2}{*}{ Parameter } & \multicolumn{3}{|c|}{ Details } \\
\hline & & $\begin{array}{l}\text { Block } \\
\text { length(n) }\end{array}$ & $\begin{array}{c}\text { Massage } \\
\text { length } \\
(\mathrm{k})\end{array}$ & $\begin{array}{l}\text { Number } \\
\text { of } \\
\text { corrected } \\
\text { error }(\mathrm{t})\end{array}$ \\
\hline 1 & BCH code & 31 & 26 & 1 \\
\hline & & 31 & 21 & 2 \\
\hline & & 31 & 16 & 3 \\
\hline & & 31 & 11 & 4 \\
\hline 2 & $\begin{array}{l}\text { Modulation } \\
\text { type }\end{array}$ & \multicolumn{3}{|c|}{$\begin{array}{c}\text { Binary phase-shift keying } \\
\text { modulation. }\end{array}$} \\
\hline 3 & $\begin{array}{l}\text { Type of } \\
\text { channel }\end{array}$ & \multicolumn{3}{|c|}{ Rayleigh fading channel } \\
\hline 4 & $\begin{array}{c}\text { Number of } \\
\text { transmitting } \\
\text { and } \\
\text { receiving } \\
\text { antenna } \\
\text { over MIMO } \\
\text { system }\end{array}$ & \multicolumn{3}{|c|}{$2 * 2$} \\
\hline
\end{tabular}

\section{Simulation Results}

In this section, we focus on BER performance and throughput on the proposed model. Table 2 demonstrates the main parameters utilizing in simulation.
Table 2.Main parameters utilizing in simulation

Fig.2 demonstrates the comparison of a BER performance of the proposed model with various lengths of $\mathrm{BCH}$ codes over the MIMO system, with a Rayleigh fading channel, respectively. $\mathrm{BCH}(31,26)$ codes having the capability to correct one error $(\mathrm{t}=1)$, Also $\mathrm{BCH}(31,21)$ correct two errors $(\mathrm{t}=2)$. $\mathrm{BCH}(31,16)$ correct three errors $(\mathrm{t}=3)$, and $\mathrm{BCH}(31,11)$ correct four error $(\mathrm{t}=4)$. The proposed model achieved enhancement in coding gain. For the BCH (31, 26) code accomplished enhancement in coding gain approximately $6.94 \mathrm{~dB}$ at BER about $\left(10^{-3}\right)$, while obtaining even better enhancement when the $\mathrm{BCH}(31,21)$ about 10.7 $\mathrm{dB}$ Furthermore, when $\mathrm{BCH}(31,16)$ nearly enhancement $13.25 \mathrm{~dB}$, and finally when $\mathrm{BCH}$ $(31,11)$ the enhancement about $16.9 \mathrm{~dB}$.

Furthermore, the proposed model achieved improvement in the performance of the MIMO system, and this improvement is maximized with an increased length of $\mathrm{BCH}$ code. Thus, it can be calculated this improvement at the $(\mathrm{SNR}=10$ $\mathrm{dB})$. For the $\operatorname{BCH}(31,26), \operatorname{BCH}(31,21)$, $\mathrm{BCH}(31,16), \quad \mathrm{BCH}(31,11)$ improvement of system about 65\%, 95\%, $100 \%, 100 \%$ ,respectively, when compared with the un-coded MIMO system 


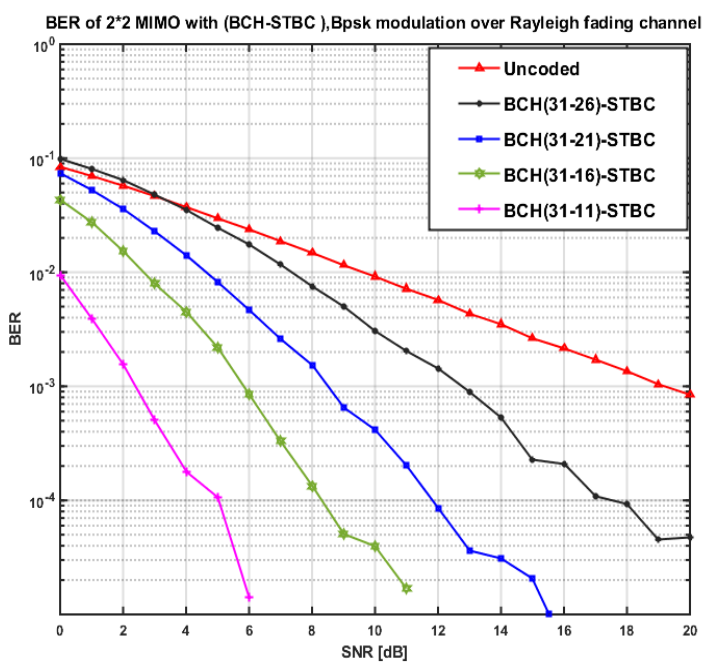

Figure2. BER comparison of combining $\mathrm{BCH}(31,26)$, $(31,21),(31,16),(31,11)$ codes with STBC code over MIMO system, with un-coded state, over Rayleigh fading channel

Fig.3 demonstrates throughput comparison of the proposed model with various lengths of $\mathrm{BCH}$ codes over the MIMO system with a Rayleigh fading channel.

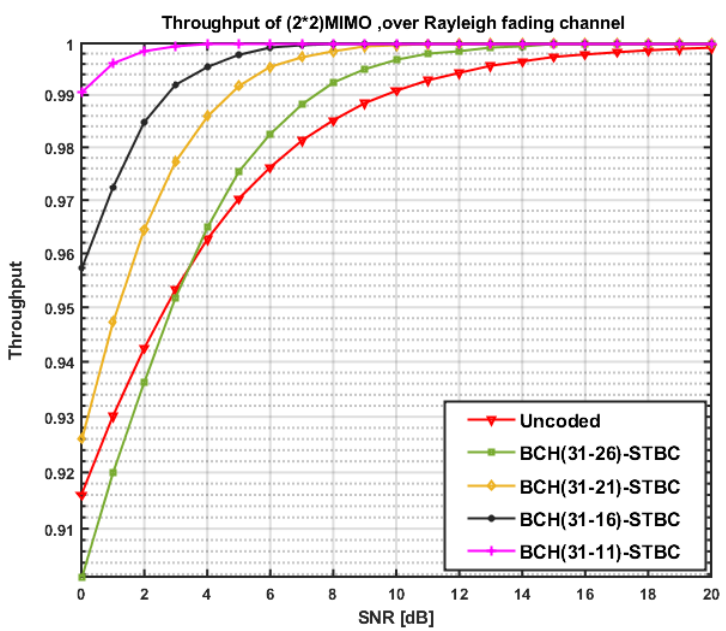

Figure 3. Throughput comparison of combining $\mathrm{BCH}$ $(31,26),(31,21),(31,16),(31,11)$ codes with STBC codes over MIMO system, with un-coded state, over Rayleigh fading channel

Typically, the enhancement of the throughput relies on the coding techniques utilizing and SNR [dB]. The throughput estimated will be as predicted proportional directly with the SNR [dB].

The (green, blue, black, pink) curves in Fig.3 indicate a comparison of throughput of the proposed model with various length of $\mathrm{BCH}$ codes utilized, with un-coded MIMO state. Thus, accomplished maximum improvement throughput at $(\mathrm{SNR} \geq 18 \mathrm{~dB}),(\mathrm{SNR} \geq 13 \mathrm{~dB})$ $(\mathrm{SNR} \geq 10 \mathrm{~dB})$, and $(\mathrm{SNR} \geq 6 \mathrm{~dB})$.respectively.

\section{Conclusions}

The main objective of several communication systems is to achieve the highest system throughput and minimize BER. In this research paper, we proposed a model combining $\mathrm{BCH}$ (n , k) code with STBC code over the MIMO system with the Rayleigh Fading channel to improve throughputs and minimize bit error rate (BER) performance. The proposed model accomplishes good performance in terms (BER, throughput, coding gain, and system improvement) compared with the un-coded MIMO system. So, better outcomes were obtained when using the BCH $(31,11)$ code in the proposed model. It achieved coding gain about $16.9 \mathrm{~dB}$ and improve system performance $100 \%$ compared with the un-coded MIMO system, also getting maximum throughput at (SNR $\geq 6 \mathrm{~dB}$ ). Moreover, all these improvements obtained by exploiting the error correction capability feature that having in $\mathrm{BCH}(\mathrm{n}, \mathrm{k})$ codes and utilizing a remarkable MIMO technique over Rayleigh fading channel. The obtained result supports the major idea and achieves the required goal.

\section{Acknowledgments}

The authors wish to thank Al-Mustansiriyah University for the support of the research work.

\section{Conflict of interest}

The authors declare that there is no conflict of interest.

\section{References}

1. Alizadeh, M., Baghersalimi, G. and Nassiri, M. (2019). "Performance evaluation of $2 \times 2$ MIMO-OFDM based radio-over-fibre system using Alamouti coding and Hammerstein equaliser", IET Communications, vol. 13, no. 9, pp. 1171-1178. 
2. Kshetrimayum, R. S. (2017). "Fundamentals of MIMO wireless communications", Cambridge University Press.

3. Khalid,M., Naqvi,S.I., Amin,Y., and Tenhunen,H. (2020). "An integrated 4G Array with mm-wave 5G MIMO Antenna for Future Mobile applications", in 2020 3rd International Conference on Computing, Mathematics and Engineering Technologies (iCoMET). IEEE, pp. 1-6.

4. Yun, S. and Qiu, L. (2015). "Supporting WiFi and LTE co-existence", in 2015 IEEE Conference on Computer Communications (INFOCOM). IEEE, pp. 810-818.

5. Thaher,R.H. (2009). "Environmental analysis of MIMO channel capacity under variable factors",ERJ. Engineering Research Journal. Menoufia University, Faculty of Engineering, vol. 3 ,no. 9 ,pp. 287-297.

6. Xiao, H. et al. (2010). "A novel JSCC framework with diversity-multiplexingcoding gain tradeoff for scalable video transmission over cooperative MIMO", IEEE transactions on circuits and systems for video technology. IEEE, vol. 20, no. 7, pp. 994-1006.

7. El Falou, A. et al. (2013) "Finite-SNR diversity-multiplexing tradeoff for Rayleigh MIMO channels", IEEE communications letters. IEEE, vol. 17, no. 4, pp. 753-756.

8. Sarwar, A., Shah, S. M. and Zafar, I. (2020) "Channel Estimation in Space Time Block Coded MIMO-OFDM System using Genetically Evolved Artificial Neural Network", in 2020 17th International Bhurban Conference on Applied Sciences and Technology (IBCAST). IEEE, pp. 703709.

9. Mike Buehrer, W. T. (2006). Code Division Multiple Access(CDMA), Synthesis Lectures on Communications. Morgan \& Claypool Publishers.
10. Shankar, P. M. (2017). "Fading and shadowing in wireless systems". Springer.

11. Popescu, S. O. and Gontean, A. S. (2011). "Performance comparison of the BPSK and QPSK Modulation Techniques on FPGA", in 2011 IEEE 17th International Symposium for Design and Technology in Electronic Packaging (SIITME). IEEE, pp. 257-260.

12. Kadhim, A. A. and Alubaidy, A. (2012) "Throughput improvement for wireless networks using MIMO network coding", in 2012 First National Conference for Engineering Sciences (FNCES 2012). IEEE, pp. 1-7.

13. Zhao, S. et al. (2013). "A concatenation scheme of Polar codes and space-time block codes in multiple-input multiple-output channels", Proceedings of the 2013 6th International Congress on Image and Signal Processing, CISP 2013, vol. 3, no. 6, pp. 1216-1220.

14. Ghayoula, E. et al. (2015) "Improving MIMO systems performances by concatenating LDPC decoder to the STBC and MRC receivers", 2015 World Symposium on Computer Networks and Information Security, WSCNIS 2015.

15. Gazi, O. (2020) "Forward Error Correction via Channel Coding", Springer .

16. Xue, T. et al. (2018). "Adaptive Spatial Modulation Combining $\mathrm{BCH}$ Coding and Huffman Coding", in 2018 IEEE 23rd International Conference on Digital Signal Processing (DSP). IEEE, pp. 1-5.

17. Alwan, M. H., Singh, M. and Mahdi, H. F. (2015). "Performance comparison of turbo codes with LDPC codes and with $\mathrm{BCH}$ codes for forward error correcting codes", in 2015 IEEE Student Conference on Research and Development (SCOReD). IEEE, pp. 556-560.

18. Lone, F. R., Puri, A. and Kumar, S. (2013). "Performance Comparison of Reed Solomon 
Code and BCH Code over Rayleigh Fading Channel", International Journal of Computer Applications, vol. 71, no. 20, pp. 23-26.

19. Lin, S. (2004). "D. Costello Error Control Coding". Pearson, Prentice Hall. 